Case Report

\title{
An Unusual Presentation of Glucose Transporter Type 1 Deficiency Syndrome
}

Saritha G. ${ }^{1}$, Bhavani S. ${ }^{2}$

${ }^{1,2}$ Assistant Professor, M.Sc. Pediatric Nursing, College of Nursing, Pondicherry Institute of Medical Sciences, Puducherry-14

*Corresponding Author : Saritha G, Assistant Professor, M.Sc. Pediatric Nursing, College of Nursing, Pondicherry Institute of Medical Sciences, Puducherry-14, Mobile-09786740206, E-Mail gsaritha@gmail.com

Received : 13.09 .2018

Review Completed : 15.11.2018

Accepted

: 21.11 .2018

Keywords : Ketogenic diet; Glucose transporter deficiency syndrome type I (GLUT1).

\begin{tabular}{|c|}
\hline Access this article online \\
\hline Quick Response Code \\
\hline
\end{tabular}

\begin{abstract}
Glucose serves as the major energy sources for developing brain. In the resting state, the adult brain can consume up to $25 \%$ of the body's total glucose supply, while in infants and children it can use as much as $80 \%$. Glucose entry into brain occurs via glucose transporter proteins GLUTS). They are five GLUTs protein. Out of five GLUTs 1 and GLUTS 3 are located in brain, with GLUTS-1 found in blood brain barrier and choroid plexus and GLUTs-3 found in neurons. Glucose transporter type 1 deficiency syndrome is an impaired glucose transport into the brain as the result of a mutation of the SLC2A1 gene leads to development of a metabolic encephalopathy of developing brain.
\end{abstract}

\section{Case Report}

We present a case of 10 days old male baby brought to hospital with the history of two episodes of jerky movements of upper and lower limbs lasted for 2 to 3 minutes with the flickering noticed since one day. The boy was the second child for third degree consanguineous parents. He was spontaneously conceived and born. Cried, soon after birth, birth weight of 2980 grams and history of admission for $48 \mathrm{hrs}$ in the neonatal intensive care unit in view of transient tachypenea of newborn. Mother's three siblings had symptoms of developmental delay with repeated attack of seizure and were treated with antiepileptic drugs. All three siblings died with 10 yrs age. This Child has a 6 yrs healthy sister. On observation the baby had HR -139 beat/min, RR-52/min, SPO2- $98 \%$, CFT- 3 Seconds, Weight - $2.987 \mathrm{~kg}$, Length - $50 \mathrm{~cm}$, Head circumference $-34 \mathrm{~cm}$ Chest circumference- $32 \mathrm{~cm}, \mathrm{CVS}-\mathrm{S} 1$ S2 heard - no murmurs Breath Sound- Normal Vesicular Breath Sounds, Abdomen - Soft Bowel Sounds Present,
CNS -Rooting Sucking reflex - good, Moro reflex - Complete and Moves all four limbs.

Initial investigation of Peripheral smear, blood gas analysis, serum Creatinine, ammonia, lactate, blood urea, cortisol, bilirubin level, liver profile and electrolyte was done. All investigations were normal. The baby was monitored for CBG - Q 6 hourly and urine ketone Q $12^{\text {th }}$ hourly. The results show Normal CBG and Urine acetone - negative. EEG was normal. TMS and urine GCMS sample were sent 'Brain MRI with lactate peaks was done which showed normal study. Then lumbar puncture was done which shows CSF/blood glucose ratio less than 0.5 .

So the baby was suspected to have Glucose transporter type 1 deficiency syndrome. Advised to stop breast feeding. He was started on ketogenic milk formula contains high fat and low $\mathrm{CHO}$. He had no episode of seizure after 48 hrs ketogenic formula. Then Repeat EEG was taken after stopping ketogenic milk formula, he developed seizure and 
developed epileptic form activity. so started ketogenic diet, Syrup levitiracetam $20 \mathrm{mg} / \mathrm{kg} /$ day-0.5 ml Q12 hourly ((100mg / $1 \mathrm{ml}$ ) and Tab pyridoxine $40 \mathrm{mg}-0$-60 mg -oral daily. then baby is gaining weight and no episode of seizure hence baby discharged with genetic counseling and follow up.

\section{Introduction}

There are two possible mechanisms by which decreased brain glucose can cause neurologic disturbance: One is decreased fuel (energy), and the other is abnormal thalamocortical maturation (development).GLUT1DS is also known as de vivo disease is a rare genetic disorder mainly affecting the CNS. Mutations in SLC2A1 are inherited in an autosomal dominant manner and $90 \%$ of cases are de novo (arising new in that individual and not inherited from either parent). The disease was first described in 1991. About 500 patients have now been recorded around the world. The incidence of glut1 is independent of gender, age, and nationality. One in 90,000 people are born with Glut 1 DS. Prevalence is as many as 7,000 people in the United States. Male and female are equally affected It is caused by a defect in the SLC2A1 gene, which encodes the glucose transporter responsible for transporting glucose from the blood to the brain across the blood-brain barrier.

\section{Discussion}

Mutations in the SLC2A1 gene generally spontaneous, though some families as autosomal dominant inheritance. GLUT1 is on rare occasions inherited as an autosomal recessive. It can alter or completely block the function of GLUT1 protein, which results, lack of energy to the brain, leading to progressive impairment of brain functions. Glucose transporter type 1 (GLUT1) facilitates the passage of glucose across the blood brain barrier. GLUT1 Deficiency Syndrome (GLUT1DS) results from impaired glucose transport into the brain will leads to development of a metabolic encephalopathy of developing brain, developmental delay and recurrent seizure etc.

1. clinical manifestations include infantile seizures (often resistant to traditional seizure medications), Psychomotor delay , Acquired Microcephaly, 90 \% affected children develop epilepsy (various types including absence, focal, generalized, myoclonic, clonic, tonic and non convulsive status $.10 \%$ of child with Glut 1 Deficiency do not have seizures and symptoms are typically less severe. nonepileptic cases still have developmental delay, intellectual delays, and movement disorders such as ataxia, alternating hemiplegia, or dystonia involuntary eye movements, spasticity, hypotonia and Behavioral problems.

\section{Diagnostic evaluation}

a) Lumbar puncture - Reduced cerebrospinal fluid (CSF) glucose (hypoglycorrhachia) in the presence of normal blood glucose due to impaired glucose transport across the blood-brain barrier.

b) Gene analysis is recommended for baby with clinical findings and low cerebrospinal fluid glucose $(<50 \mathrm{mg} / \mathrm{dl}$. Both partial and complete SLC2A1 gene deletions have been identified.

c) EEG findings -Shows a normal interictal EEG. It can present with multifocal spike discharges on EEG, underlying infantile focal seizures (non-generalized) that may include eye movements and cyanotic spells

d) Magnetic resonance imaging (MRI) - Demonstrate diffuse atrophy

e) Positron emission tomography (PET)- a global decrease in glucose uptake in the cortex, more severe in the mesial temporal regions and thalamus and less marked in the basal ganglia.

3. Management Early diagnosis is important because it allows prompt initiation of treatment to prevent developmental delay in children .Ketogenic diet- is a highfat, adequate-protein, low-carbohydrate diet and Genetic counseling .Pharmacological agents known to impair GLUT1 function, e.g. caffeine, Phenobarbital, diazepam, methylxanthines, tricyclic antidepressants and alcohol should be avoided.Alpha lipoic acid supplementation is recommended to improve glucose transport in muscle cells via mobilization of the GLUT4 transporter from 
intracellular pools. Occupational, physical, and speech/language therapies are standard for most patients, especially in childhood. Follow-up visits are needed to monitor closely.

\section{Nursing care}

- Advised The mother to stop breast feeding

- Feed the baby with KD Formula

- Institute Seizure Precautions

$\checkmark$ Place the baby on the floor.

$\checkmark$ Turn gently onto one side to help breathe.

$\checkmark$ Clear the area around the baby of anything hard or sharp to preventinjury.

$\checkmark$ Put something soft and flat, like a folded jacket, under his or her head.

$\checkmark$ Loosen cloths or anything around the neck that may make it hard to breathe.

$\checkmark$ Stay with the baby until the seizure ends and he or she is fully awake.

- Genetic Counseling

References

1. Pyush Gupta,PSN Menon, Siddarth Ramji, Rakesh Lodha. Post Graduate (2018) Text Book Of Pediatrics, Jaypee health sciences publisher New Delhi ; Volume-3, 2197.

2. David.G Nichos.et al (2012).Roger's Text Book of Pediatric Intensive Care. $4^{\text {th }}$ edition, Lippincott Williams and Wilkins; 821.

3. Janet M. Rennie, (2012) Rennie and Roberton's Text Book of Neonatology, $5^{\mathrm{TH}}$ Edition, Churchill Livingstone Elsevier; 786.

4. Colin.D .Rudolph, Abraham M Rudolph, George E Lister, Lewis R First, Anne A Gershon.(2010), Rudolph's Pediatrics, $22^{\text {nd }}$ edition, Mc Graw Hill Medical publishers : 612
- Continuous Oral care helps to Gingival Hyperplasia

- Growth and development monitoring and early initiation of stimuli

- Follow up

\section{Conclusion}

The prognosis of child with GLUT-1 DS depends on the underlying genetics, early initiation of treatment with a ketogenic diet and Childs follow up. Long term follow up is needed as they baby 20 days old. So the prognosis of this baby cannot be predicted at this juncture. However similar case presentation was found in journal of pediatric neurology by akiko ohshire et al -3 yrs old child with GLUT1 DS presenting with episodic ataxia. They concluded that after giving ketogenic diet, reduced the frequency of seizure attacks, increased head growth and his psychomotor and language skills have begun developing. (6) Although long-term monitoring and follow up is needed to the see the effectiveness of therapy

5. Akiko Ohhshire a., journal of pediatric oncology, 3 yrs old boy with GLUT1 DS presenting with episodic ataxia 2014, Elsevier publication page no 50.

6. Lukyanova EG, Sushko LM, Ayvazyan SO, Osipova KV, Pyreva EA Sorvacheva TN , Zhilina SS, Kozhanova TV. Glucose Transporter Type 1 Deficiency Syndrome (GLUT1) and using Ketogenic Diet in Treatment of De Vivo Disease: Electronic Journal of Biology, 2017, Vol.13 (4): 330 337

7. Assuma bee .Text book of pediatrics nursing care plan, $2^{\text {nd }}$ edition jaypee brothers $416-419$ 